\title{
Concurrent Structural and Single Nucleotide Variation Resulting from a Single Replication-Based Mechanism
}

The genomes of patients in which a genetic disorder is suspected are generally examined for single nucleotide variants (SNVs), for instance, by targeted gene, exome, or whole-genome sequencing. If the disorder of the patient is deemed to be complex instead of likely fitting the mendelian paradigm, a genome-wide search for structural genome variation (SGV) may be initiated. In this way, a conceptual dichotomy between SNVs and SGVs arises. However, there is no compelling mechanistic reason that SNVs and SGVs are mutually exclusive. The conceptual dichotomy may be rooted in technical limitations of the used technology rather than in the nature of the human genome. Recent studies using technologies that allow for SNVs and SGVs to be detected concurrently, a so-called orthogonal approach, have turned up coexisting SNVs and SGVs.

To investigate the fate of dicentric chromosomes after telomere crisis, Maciejowski et al. [2015] studied an hTERT expressing RPE-1 retinal pigment epithelial cell line in which the $\mathrm{Rb}$ and $\mathrm{p} 53$ pathways were inactivated. After deprotecting the telomeres, dicentric chromosomes were formed. These were spontaneously fragmented and rejoined, which was accompanied by formation of clusters of SNVs. These findings were reminiscent of a breakage-fusion-bridge (BFB) cycle followed by chromothripsis combined with kataegis. During a BFB cycle, a chromatin bridge is formed, which is subsequently degraded by the TREX $13^{\prime} \rightarrow 5^{\prime}$ exonuclease. The authors found that this involved a novel form of chromothripsis [Maciejowski et al., 2015]. In contrast to micronucleus-based chro- mothripsis, the BFB-based process yields both losses and gains, which are located within a single chromosome arm [Poot, 2018]. Remarkably, the chromatin bridges became covered with replication protein A. This indicates that they contain extended stretches of single-stranded DNA, a target substrate of APOBEC enzymes [Roberts et al., 2012]. These cytosine deaminases convert clusters of cytosine to thymidine or uracil in long stretches of singlestranded DNA formed in the vicinity of double-strand breaks and stalled DNA replication forks [Roberts et al., 2012]. The clusters found by Maciejowski et al. [2015] were located within $1-2 \mathrm{~kb}$ of the genomic breakpoints associated with the chromothripsis rearrangements and showed a preponderance of $\mathrm{C}>\mathrm{T}$ and $\mathrm{C}>\mathrm{G}$ mutations withina TpCcontext, which is a signature of APOBEC $3 \mathrm{~A} / \mathrm{B}$ activity [Roberts and Gordenin, 2014]. In addition, the mutation clusters were processive, which indicates that the damage occurred on a single strand of DNA. However, this study does not allow discerning how the chromosomal fragments were joined.

By whole-genome sequence analyses of clones of cells that had survived telomere crisis, an unexpected mechanism of chromosome rejoining was unveiled recently [Cleal et al., 2019]. The authors investigated the consequences of a transit through telomere crisis in cells that were deficient for NHEJ. They used HCT116 cell lines, which were either $L I G 4^{-/-}$, a double $L I G 3^{-/-}: L I G 4^{-/-}$, a LIG3 knockout line with a supra-normal complementa-

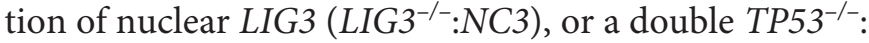
$L I G 3^{-/-}$knockout. The authors had previously shown that

\section{KARGER}

(c) 2019 S. Karger AG, Basel 
HCT116 LIG3 ${ }^{-/-}$cells are unable to escape telomere crisis, but the additional knockout of TP53 in $\mathrm{LIG}^{-/-}$cells permitted survival and, thus, sequencing of post-telomerecrisis samples. The authors found highly complex chains of SGVs, consisting of losses, gains, inversions, and translocations, reminiscent of chromoplexy or chromoanasynthesis [Liu et al., 2011; Baca et al., 2013]. These findings are inconsistent with genome shattering followed by NHEJ-mediated repair. Instead, the findings are more consistent with a replicative process initiated directly or indirectly by dysfunctional telomeres. This process is based on microhomology and error-prone DNA polymerases and involves template switching within localized genomic regions. The latter would eventually lead to a prevalence of copy number gains. This model is akin to the hypothesis of replication-based mechanisms driving complex rearrangements [Lee et al., 2007; Carvalho and Lupski, 2016]. Thus, telomere dysfunction triggering BFB cycles would account for large terminal deletions in some samples and raised numbers of copy number variations in others. Following breakage, the free chromosome ends are processed for repair, creating a $3^{\prime}$ overhang. Since, NHEJ is not available to perform repair, the $3^{\prime}$ overhang may invade nearby homologous sequences. Thus, a displacement loop is formed, which subsequently triggers error-prone break-induced repair [Malkova and Ira, 2013]. This constellation could also arise directly from short dysfunctional telomeres, with a 3 ' overhang capable of strand invasion. Then the break-induced repair machinery progresses towards the centromere, which may lead to template switching and mutagenesis. This, in turn, accounts for copy number profiles consisting of fold-back inversions and inverted fragments, and for chaining of duplicated segments upstream of a palindromic sequence. In all affected chromosomal segments, clusters of mutations reminiscent of kataegis were found [Sakofsky et al., 2014].

These observations were further extended by an indepth study of SNVs accompanying SGV formation in patients with Potocki-Lupski syndrome (MIM 610883) or Smith-Magenis syndrome (MIM 182290) due to de novo duplication or deletion at 17p11.2 [Beck et al., 2019]. The authors used both molecular cytogenetic methods and short- and long-read DNA sequencing. Combination of both kinds of methods, the so-called "orthogonal DNA sequencing approach" proved to be essential in order to unveil all possible types of SNVs and SGVs. Deep sequencing of DNA flanking the 17p11.2 SGVs showed a high SNV rate in cis with the SGVs. The affected DNA strand extended up to $1 \mathrm{Mb}$ from the SGV breakpoint junctions, and the SNVs tended to occur within genes. The SNVs at these nonrecurrent 17p11.2 SGVs result in a 5- to 50-fold higher SNV mutation rate than that observed with recurrent SGVs. This is consistent with errorprone microhomology-mediated break-induced repair (MMBIR) and contrasts with regular DNA replication of the region in healthy individuals. Nonrecurrent SGVs occur throughout the genome, and several of them have been shown to be accompanied by de novo SNVs in close proximity to the breakpoint junctions [Carvalho et al., 2013; Abyzov et al., 2015; Beck et al., 2015; Brandler et al., 2016; Yuen et al., 2016]. In one study, indels attributable to polymerase slippage were seen within a few $\mathrm{kbp}$ of a junction, as has been described before [Carvalho et al., 2013]. In addition, clusters of kataegis-like mutations, possibly due to deamination of single-stranded DNA, were found within $1 \mathrm{Mb}$ of the breakpoint junctions [Sakofsky et al., 2014]. Finally, the authors saw widely distributed base substitutions of all types, which may have resulted from MMBIR [Beck et al., 2019]. In conclusion, the authors state that replication-based repair mechanisms, such as MMBIR, may produce SGVs in conjuction with long tracts of SNVs within the de novo synthesized DNA strand. Clearly, de novo SNV mutation rates can vary in the genome, and hypermutation may be regionally restricted.

The studies discussed above not only shatter the conceptual dichotomy between SNVs and SGVs, they also stress that a "one size fits all" method to detect all clinically relevant mutations is currently not available [Hochstenbach et al., 2018]. To the contrary, we will have to rely on orthogonal, and mutually complementary, approaches in order to obtain a comprehensive picture of the mutational spectrum underlying human genetic disorders, which we need in clinical decision-making.

Martin Poot 
Beck CR, Carvalho CMB, Akdemir ZC, Sedlazeck FJ, Song X, et al: Megabase length hypermutation accompanies human structural variation at 17p11.2. Cell 176:1310-1324 (2019).

- Brandler WM, Antaki D, Gujral M, Noor A, Rosanio G, et al: Frequency and complexity of de novo structural mutation in autism. Am J Hum Genet 98:667-679 (2016).

-Carvalho CM, Lupski JR: Mechanisms underlying structural variant formation in genomic disorders. Nat Rev Genet 17:224-238 (2016).

-Carvalho CM, Pehlivan D, Ramocki MB, Fang P, Alleva B, et al: Replicative mechanisms for CNV formation are error prone. Nat Genet 45:1319-1326 (2013).

-Cleal K, Jones RE, Grimstead JW, Hendrickson EA, Baird DM: Chromothripsis during telomere crisis is independent of NHEJ, and consistent with a replicative origin. Genome Res 29:737-749 (2019).
Hochstenbach R, van Binsbergen E, SchuringBlom H, Buijs A, Ploos van Amstel HK: A survey of undetected, clinically relevant chromosome abnormalities when replacing postnatal karyotyping by whole genome sequencing. Eur J Med Genet, E-pub ahead of print (2018).

Lee JA, Carvalho CM, Lupski JR: A DNA replication mechanism for generating nonrecurrent rearrangements associated with genomic disorders. Cell 131:1235-1247 (2007).

Liu P, Erez A, Nagamani SC, Dhar SU, Kołodziejska KE, et al: Chromosome catastrophes involve replication mechanisms generating complex genomic rearrangements. Cell 146:889-903 (2011).

Maciejowski J, Li Y, Bosco N, Campbell PJ, de Lange T: Chromothripsis and kataegis induced by telomere crisis. Cell 163:1641-1654 (2015).

Malkova A, Ira G: Break-induced replication: functions and molecular mechanism. Curr Opin Genet Dev 23:271-279 (2013).
Poot M: Genes, proteins, and biological pathways preventing chromothripsis. Methods Mol Biol 1769:231-251 (2018).

Roberts SA, Gordenin DA: Hypermutation in human cancer genomes: footprints and mechanisms. Nat Rev Cancer 14:786-800 (2014).

Roberts SA, Sterling J, Thompson C, Harris S, Mav D, et al: Clustered mutations in yeast and in human cancers can arise from damaged long single-strand DNA regions. Mol Cell 46: 424-435 (2012).

Sakofsky CJ, Roberts SA, Malc E, Mieczkowski PA, Resnick MA, et al: Break-induced replication is a source of mutation clusters underlying kataegis. Cell Rep 7:1640-1648 (2014).

Yuen RK, Merico D, Cao H, Pellecchia G, Alipanahi B, et al: Genome-wide characteristics of de novo mutations in autism. NPJ Genom Med 1:160271-1602710 (2016). 